\title{
Outsourcing predictive biomarker testing in non-small cell carcinoma: a personal view of pathologists
}

\author{
Luka Brcic $^{1}$, Izidor Kern ${ }^{2}$ \\ ${ }^{1}$ Diagnostic and Research Institute of Pathology, Medical University of Graz, Graz, Austria; ${ }^{2}$ Cytology and Pathology Laboratory, University Clinic \\ of Respiratory and Allergic Diseases, Golnik, Slovenia \\ Correspondence to: Luka Brcic. Diagnostic and Research Institute of Pathology, Medical University of Graz, Neue Stiftingtalstrasse 6, 8010 Graz, \\ Austria. Email: luka.brcic@medunigraz.at.
}

Submitted Feb 19, 2020. Accepted for publication Jul 03, 2020.

doi: 10.21037/tlcr-20-297

View this article at: http://dx.doi.org/10.21037/tlcr-20-297

\section{Introduction}

Lung cancer is one of the most common cancers and has the highest mortality rate among all cancer types (1). The vast majority of lung cancers belong to the non-small cell carcinoma (NSCLC) histological type group (85\%) and small-cell lung carcinoma (15\%). Among NSCLC, the most frequent histological type is adenocarcinoma, which accounts for more than half of all NSCLC cases (2).

Since the discovery of EGFR and ALK oncogene driver mutations with consequent pivotal clinical trials $(3,4)$, we have witnessed a huge progress in biomarker-driven targeted therapies in patients diagnosed with lung adenocarcinoma in advanced stage of disease, which resulted in significantly improved progression-free and overall survival rate. Predictive biomarker testing to detect targetable genetic abnormalities became standard of care for patients with advanced NSCLC $(5,6)$. Currently, international guidelines recommend testing for the following predictive biomarkers: mutations in the kinase domain of $E G F R$ (exons 18-21), BRAF V600E mutation, ALK and ROS1 gene rearrangements, and PD-L1 expression in tumor cells (7). The number of predictive biomarkers is expanding (HER2, MET, RET, NTRK) as new targeted therapies are becoming available, but recommended turnaround time remains the same (10 working days since the arrival of the sample in molecular pathology laboratory) (7). Algorithms to detect targetable genetic abnormalities changed from single biomarker testing, either in a step-by-step approach or a parallel one, towards one-step multi-gene panel testing. The later one uses next generation sequencing
(NGS) technology, currently the standard molecular testing platform for NSCLC. Immunohistochemistry (IHC) is also a well-recognized and widely available technology to detect some predictive biomarkers. Abnormal ALK protein expression is detected by IHC efficiently and is an approved method of biomarker determination according to the international guidelines (7). IHC is also a valuable screening method to search for BRAF, ROS1 and NTRK genetic abnormalities that, in a case of a positive reaction, needs confirmation, usually by NGS. IHC is the only method available to evaluate PD-L1 expression, a predictive biomarker for immune checkpoint inhibitor therapy in NSCLC.

Predictive biomarker testing for NSCLC is challenging at current time due to the quality and quantity of tumor specimens available, lack of education for pathologists, pathology residents, as well as medical laboratory technologists that are performing the testing. Lung cancer patients in advanced stage of disease undergo less invasive diagnostic procedures and often only tiny tumor specimens are obtained. Pathologists and medical laboratory technologists have to deal with small biopsies and cytological specimens, because scant tumor specimens have major impact on laboratory processing. An optimal balance between accurate histologic typing and tissue sparing is needed to allow consecutive predictive biomarker testing. The diagnostic part of the pathology is in most cases performed in-house, while predictive biomarker testing might be outsourced. The decision where to perform predictive biomarker testing, in-house or in a referral laboratory, should be based on several considerations: 
technology and staff available, turnaround time, expertise, quality assurance, cost effectiveness, clinical validation, minimum number of tests, and legal concerns. Regardless of the decision, it is very important not to waste the tumor samples in the pre-analytical phase. The optimal approach includes complete predictive biomarker testing in one place in order to reduce handling of tumor specimen.

\section{In-house predictive biomarker testing}

The best option for patients and the clinicians involved in therapy decisions is to have access to a pathology laboratory, which can perform complete predictive biomarker testing on its own. The advantages are clear- there is no waste of time by sending material to a referral laboratory, no additional time needed for extra paperwork, and no legal issues since pathologists who have signed the report are also responsible for its interpretation and clarification in multidisciplinary tumor-board meetings. Furthermore, pathologists who made original diagnosis have all the information about patient samples, including availability of another sample, if for example, there is not enough tumor content necessary for all predictive biomarkers testing in the selected sample. In case that tumor tissue is very limited, pathologists can discuss with clinicians directly in which sequence to perform molecular tests or select predictive biomarkers important to test first. When several tumor specimens from the same patient are available, pathologists can triage them for diagnostic and molecular testing, choosing from available methods and platforms. The possibility of additional tumor sampling can also immediately be discussed. Advantages for in-house analysis include also the possibility of maintaining clinical registries, with continuous clinical validation of results/methods.

A drawback of in-house molecular pathology is that the infrastructure is expensive, and in low-volume laboratories without adequate sample numbers, some methods, like NGS, are not economically feasible. To use consumables efficiently, the turnaround time (TAT) or analysis might be longer in order to fill the batch/plate. Considering these drawbacks, mentioned laboratories could decide to go for "plug-and-play" systems, which are very robust, reliable, and easy to use, providing in general satisfactory results $(8,9)$. These systems usually have rather short "handson" time and, unfortunately, consume more tumor tissue for single gene analyses than NGS for multiple gene analyses. This is of course not an issue with resection specimen, where tumor tissue is abundant, but might be an issue in small biopsies and cytological samples, where the amount of tumor cells is limited. From the perspective of smaller laboratories (multiple)-single-gene testing might be satisfactory. However, we are witnessing very fast development of new targeted drugs, resulting in a need to re-test the samples of the patients who are eligible for these new therapies, if certain mutations are present. In many cases, there is no enough tumor left in original small biopsy or cytological specimens to perform these additional predictive biomarkers tests. In addition, the patients are very often in a clinical state that they are unable to endure additional new biopsies. The major advantage of NGS is that in one step a gene panel containing relevant predictive biomarkers for approved therapies, is tested simultaneously. Most of the panels include also genes important for therapies which are currently in clinical studies. A very recent example is biomarker driven targeted therapy against the KRAS mutation $\mathrm{G} 12 \mathrm{C}$, which at this point looks very promising (10). If patient tumor samples were tested in last few years by NGS, they would have these mutation results already included. However, if patients were tested only for currently approved targeted therapies, KRAS would not have been tested routinely, and all patients potentially eligible for this therapy need to be retested, requiring enough tumor tissue still to be available. Another issue in low-volume laboratories, regardless of the method(s) they are using, is adequate quality assurance, which is more difficult to achieve and maintain (11). Furthermore, some gene mutations, like fusions, are more easily and reliably detected using RNA-based panels for NGS, and currently there are no single gene analysis providing similar results. DNA-based panels for multiple gene testing, which provide analysis of gene fusions, will not be able to detect all fusions, especially in terms of detecting rare mutations.

Larger pathology laboratories (most commonly based within Universities, or associated with a University Hospital Center) are high-volume laboratories, with fast turnover of tumor samples, higher number of experienced staff, including not only pathologists, but also molecular biologists and bioinformaticians, who play an important part in predictive biomarker testing. Higher sample numbers make the whole process more cost-efficient since consumables are efficiently used with multiple patient samples per run. Furthermore, molecular biologists and bioinformaticians analyzing many samples per week have huge experience, and can recognize potential pitfalls, problematic results/readings and can, therefore, "override" automatic analyses by looking into the raw data, answering 
sometimes crucial questions for the therapy decisions. In addition, there is always back-up technology available to solve problematic cases or in situations when the primary testing technology is not available. All "unusual" findings can directly be discussed between the pathologists in charge of signing out the report and the molecular biologists responsible for data acquisition and analysis. The legal issues concerning responsibility for the report are way less complicated, and incorporating testing results in pathology report and making comments is much easier. Legal issues may vary from one country to another, and may involve not only pathologist, but also oncologist, and institutions (both internally and externally). However, this discussion is out of the scope of this paper. To sum it up: pathologists are crucial not only for diagnosis and adequacy assessment of the obtained material for predictive testing, but also for the interpretation of results of these tests and active participation in discussion of potential therapy options during multidisciplinary board meetings ("tumor-boards").

\section{Outsourcing predictive biomarker testing}

It is clear that there are several important points to have in mind when thinking about outsourcing predictive biomarker testing: financial issues, quality and expertise, data management and security, and as mentioned before, legal issues (12).

For low-volume laboratories to invest in equipment and staff for NGS is not a viable option. Moreover, IHC using approved diagnostic kits (e.g., ALK, PD-L1) is expensive and platform dependent. Smaller systems for single gene analyses are a good option, as TAT is shorter and they are easier to handle, however with all limitations in terms of tumor specimen wasting and cost-effectiveness. When tumor tissue is limited, it is in the interest of the patient to send the sample to a laboratory with an NGS platform. Indeed, it is the right of the patients, directly connected with their future therapy options and survival. Today, when there are many predictive biomarkers recommended for routine testing in NSCLC, single-gene analyses are not cost efficient and consume significantly more tumor-tissue. According to international recommendations, multiplegene testing is preferred over single-gene analysis (7). As mentioned before, new targeted therapies are already in advanced clinical studies, and it is only matter of time when additional genes need to be tested.

When outsourcing, it is important to be certain about the expertise of the professionals performing analysis, and about the adequate quality and TAT of performed tests. Overall TAT might be adequate or longer than recommended, hampering further therapy decisions (13-15). Data management and security are today strictly regulated, and this should be no issue. However, availability of "raw" sequencing data should also be a standard in order to provide pathologists in charge of patient adequate preparation for discussion about therapy options.

There are two possibilities. One is to send samples to the high-volume pathology department, which has expert pathologist and medical laboratory technologists, who can provide fast and reliable analyses. In this setting, dedicated pathologists will re-evaluate the sample, and in case of any questions call the referring pathologists/oncologists. Samples containing only a low percentage of tumor cells can still be analyzed with a caveat in case of negative results. Furthermore, the possibility of direct communication with the pathologist who signed out the molecular pathology report is also important to clarify possible questions prior (or during) tumor board meetings. Another option is sending tumor sample to one of the commercial laboratories. These are usually laboratories specialized in molecular analyses only. The referring pathologist/oncologist should send paraffin block(s) or pre-cut slides with adequate tumor tissue content. Sending paraffin blocks is today against the policy of many pathology departments, and requested number of slides can be as high as 20 , which is usually more than used/needed by university/hospital based centers. In the case of tumors with low tumor cell content (e.g., $<10 \%$ ), samples will be rejected up-front and not accepted for analysis. Overall TAT are usually a bit longer, mainly due to the shipment procedures. The number of genes tested is higher, but there is usually no access to "raw" sequencing data. Results and their interpretation as well as links with ongoing clinical trials and potential off-label medication, although impressive in scope and content, is also potential cause of problems for patients and clinicians. Patients receive information about possibilities of off-label therapy, and currently running clinical trials all over the world (for some genes several hundreds of trials), without a realistic chance for the patient to be included, or to receive the offlabel drug. Patients are usually not aware that, if included in a clinical trial, they do not always receive "the drug", which is dependent on the study arm in which the patient will be included. Clinicians on the other hand have problems in explaining to patients why they cannot receive this off-label therapy, or why there is no chance for them to be included in clinical trial. Although this information might be 
beneficial for a certain number of patients (also depending on the country/region), for most patients it will cause unrealistic expectations and, consequently, frustrations. Another interesting point is that different analysis software programs frequently provide different clinical trial lists and potential off-label drug usage based on the same mutations detected. The next problematic issue might be integration of reports lacking raw data and knowledge of the analytical process itself into regular multidisciplinary boards, which is again related to country-specific legal issues. Furthermore, interpretation of data might also be problematic, both when these are received from provider of results as mentioned above, or when it is done in low volume center without adequate experience. Finally, the price for these analyses might be higher than the price of NGS analysis at University/hospital center-based pathology institutes.

\section{Conclusions}

Predictive biomarker testing in patients with NSCLC is today a standard of care, and is performed using IHC and molecular analysis. In our opinion, if complete testing cannot be performed in low-volume laboratories without enough resources (where original diagnosis was made), it should be sent to the laboratory with adequate technology, educated staff, short turnaround time and successful participation in external quality assessment (EQA). Furthermore, these laboratories must have expertise and perform regular quality assurance, with clinical validation. Acquired data should always be completely and fully available to pathologists who are legally responsible for the report as well as the incorporation of molecular analysis data in pathohistological analysis and its interpretation.

\section{Acknowledgments}

Authors are grateful to Prof. Gerald Höfler for his help in proof reading this manuscript.

Funding: None.

\section{Footnote}

Provenance and Peer Review: This article was commissioned by the Guest Editor (Helmut H. Popper) for the series "New Developments in Lung Cancer Diagnosis and Pathological Patient Management Strategies" published in Translational Lung Cancer Research. The article was sent for external peer review organized by the Guest Editor and the editorial office.

Conflicts of Interest: Both authors have completed the ICMJE uniform disclosure form (available at http:// dx.doi.org/10.21037/tlcr-20-297). The series "New Developments in Lung Cancer Diagnosis and Pathological Patient Management Strategies" was commissioned by the editorial office without any funding or sponsorship. LB reports grants, personal fees and non-financial support from AstraZeneca, personal fees from BMS, personal fees and non-financial support from MSD, personal fees from Merck, personal fees from Boehringer-Ingelheim, personal fees from Eli Lilly, personal fees from Takeda, personal fees and non-financial support from Pfizer, personal fees and non-financial support from Roche, outside the submitted work. IK reports personal fees from AstraZeneca, personal fees from MSD, personal fees from Boehringer-Ingelheim, personal fees from Roche, outside the submitted work. The authors have no other conflicts of interest to declare.

Ethical Statement: The authors are accountable for all aspects of the work in ensuring that questions related to the accuracy or integrity of any part of the work are appropriately investigated and resolved.

Open Access Statement: This is an Open Access article distributed in accordance with the Creative Commons Attribution-NonCommercial-NoDerivs 4.0 International License (CC BY-NC-ND 4.0), which permits the noncommercial replication and distribution of the article with the strict proviso that no changes or edits are made and the original work is properly cited (including links to both the formal publication through the relevant DOI and the license). See: https://creativecommons.org/licenses/by-nc-nd/4.0/.

\section{References}

1. Global Cancer Observatory. Available online: https:// gco.iarc.fr/today/data/factsheets/cancers/15-Lung-factsheet.pdf

2. Tumours of the lung. In: Travis WD, Brambilla E, Burke AP, et al. (eds). WHO Classification of Tumours of the Lung, Pleura, Thymus and Heart. Lyon: IARC, 2015:9-152.

3. Mok TS, Wu YL, Thongprasert S, et al. Gefinitib or Carboplatin-Paclitaxel in Pulmonary Adenocarcinoma. N Engl J Med 2009; 361:947-57.

4. Soda M, Choi YL, Enomoto M, et al. Identification of the 
transforming EML4-ALK fusion gene in non-small-cell lung cancer. Nature 2007; 448:561-6.

5. Planchard D, Popat S, Kerr K, et al. Metastatic non-small cell lung cancer: ESMO Clinical Practice Guidelines for diagnosis, treatment and follow-up. Ann Oncol 2018;29:iv192-iv237.

6. NCCN version 4.2020. Available online: https://www. nccn.org/professionals/physician_gls/pdf/nscl.pdf

7. Lindeman NI, Cagle PT, Aisner DL, et al. Updated Molecular Testing Guideline for the Selection of Lung Cancer Patients for Treatment with Targeted Tyrosine Kinase Inhibitors: Guideline From the College of American Pathologists, the International Association for the Study of Lung Cancer, and the Association for Molecular Pathology. Arch Pathol Lab Med 2018;142:321-46.

8. Evrard SM, Taranchon-Clermont E, Rouquette I, et al. Multicenter Evaluation of the Fully Automated PCRBased Idylla EGFR Mutation Assay on Formalin-Fixed, Paraffin-Embedded Tissue of Human Lung Cancer. J Mol Diagn 2019;21:1010-24.

9. Van Haele M, Vander Borght S, Ceulemans A, et al. Rapid clinical mutational testing of KRAS, BRAF and EGFR: a prospective comparative analysis of the Idylla technique

Cite this article as: Brcic L, Kern I. Outsourcing predictive biomarker testing in non-small cell carcinoma: a personal view of pathologists. Transl Lung Cancer Res 2020;9(5):2194-2198. doi: 10.21037/tlcr-20-297 with high-throughput next-generation sequencing. J Clin Pathol 2020;73:35-41.

10. Nagasaka M, Li Y, Sukari A, et al. KRAS G12C Game of Thrones, which direct KRAS inhibitor will claim the iron throne? Cancer Treat Rev 2020;84:101974.

11. Tack V, Schuuring E, Keppens C, et al. Accreditation, setting and experience as indicators to assure quality in oncology biomarker testing laboratories. Br J Cancer 2018;119:605-14.

12. Available online: https://www.bhnco.com/Resources/ outsourcing-medical-diagnostics.html

13. DiStasio M, Chen Y, Rangachari D, et al. Molecular Testing Turnaround Time for Non-Small Cell Lung Cancer in Routine Clinical Practice Confirms Feasibility of CAP/IASLC/AMP Guideline Recommendations: A Single-center Analysis. Clin Lung Cancer 2017;18:e349 56.

14. Vigliar E, Malapelle U, Bellevicine C, et al. Outsourcing cytological samples to a referral laboratory for EGFR testing in non-small cell lung cancer: does theory meet practice? Cytopathology 2015;26:312-7.

15. Lambros L, Uguen A. Toward a Molecular Diagnosis in a Single Day for Patients With Advanced Non-small-cell Lung Cancer. Clin Lung Cancer 2018;19:e537-8. 